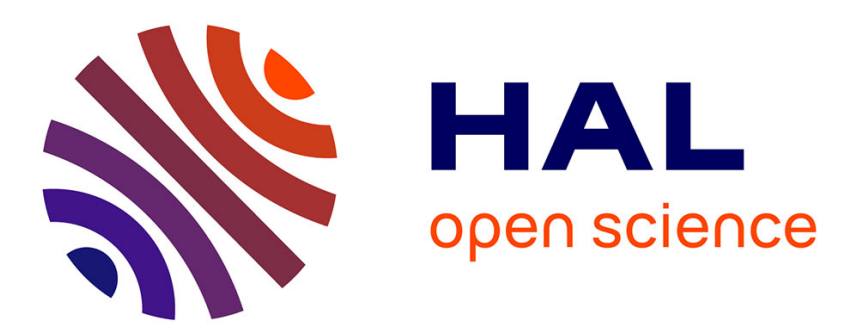

\title{
A novel tactile feedback system with on-line texture decoding and direct-texture-feedback
}

\author{
Kuniharu Sakurada, Gowrishankar Ganesh, Wenwei Yu
}

\section{To cite this version:}

Kuniharu Sakurada, Gowrishankar Ganesh, Wenwei Yu. A novel tactile feedback system with on-line texture decoding and direct-texture-feedback. ICRA 2021 - 38th IEEE International Conference on Robotics and Automation, May 2021, Xi'an, China. pp.12946-12953, 10.1109/ICRA48506.2021.9561724. hal-03042579

\section{HAL Id: hal-03042579 \\ https://hal.science/hal-03042579}

Submitted on 6 Dec 2020

HAL is a multi-disciplinary open access archive for the deposit and dissemination of scientific research documents, whether they are published or not. The documents may come from teaching and research institutions in France or abroad, or from public or private research centers.
L'archive ouverte pluridisciplinaire $\mathbf{H A L}$, est destinée au dépôt et à la diffusion de documents scientifiques de niveau recherche, publiés ou non, émanant des établissements d'enseignement et de recherche français ou étrangers, des laboratoires publics ou privés. 


\title{
A novel tactile feedback system with on-line texture decoding and direct-texture-feedback
}

\author{
Kuniharu Sakurada ${ }^{1,4}$, Gowrishankar Ganesh ${ }^{2}$, and Wenwei $\mathrm{Yu}^{3,4}$
}

\begin{abstract}
Tactile perception on our fingers is a key sensory feedback that enables us to perceive and explore our world using our hands as probes, and is essential for efficient gripping and manipulation of objects. A tactile feedback system can therefore greatly improve the quality of life of individuals with partial or complete sensory loss like during stroke, or with artificial limbs after an amputation. However, most existing tactile texture feedback technologies suffer from two constraints. First, texture decoding and texture feedback have been traditionally examined separately and not as parts of the same problem, and second, texture information has been popularly fed back using sensory modality other than tactile itself. In this study, we propose a prototype on-line direct-texture decoding and feedback system in which the texture touched by a user is decoded using an accelerometer attached to the finger. The feedback is realized by rubbing the user's skin instantaneously with the actual material that, and at the speed at which, the user swipes. The efficacy of the proposed system was tested in two user experiments with five test materials. The results and the corresponding hints for future improvements are discussed.
\end{abstract}

\section{INTRODUCTION}

Tactile sensory information is fundamental to our perception of our environment, and is a major determinant of our hand functions [1], [2], including manipulation and grasping. Touch and tactile feedback are also crucial during our interaction with other humans individuals [3], [4]. Tactile feedback training and restoration are therefore important for individuals who have suffered from a partial or complete tactile feedback, such as stroke patients, and amputees [5], [6]. Upper limb amputees usually compensate the absence of tactile feedback with vision, but vision-dependent task execution causes excessive cognitive load on individuals [7]. On the other hand, substituting stimuli for tactile feedback is known to help the recovery of original tactile functions that have been impaired or lost [8], [9].

Existing tactile texture feedback technologies, such as [10], suffer from several limitations. First, cost and applicability to human subjects remain the key hurdles for decoding

\footnotetext{
* This work was supported by JSPS KAKENHI Grant Number 17 H02129 to Wenwei YU.

${ }^{1}$ Kuniharu Sakurada is with Graduate School of Science for Open and Environmental Systems, Center for Information and Computer Science, University of Keio, Kohoku-ku, Yokohama, 2238522, Kanagawa, Japan. kh. sakurada@keio.jp

${ }^{2}$ Gowrishankar Ganesh is with the UM-CNRS Laboratoire d'Informatique, de Robotique et de Microelectronique de Montpellier (LIRMM) , 161 Rue Ada, 34095 Montpellier Cedex 5, France. ganesh.gowrishankar@lirmm. fr

${ }^{3}$ Wenwei $\mathrm{Yu}$ is with Center for Frontier Medical Engineering, Inage, 2638522, Chiba, Japan. yuwillefaculty. chiba-u.jp

${ }^{4}$ Kuniharu Sakurada and Wenwei Yu are with Graduate School of Science and Engineering, Divisions of Fundamental Engineering, Department of Medical Engineering, University of Chiba, Inage, 2638522, Chiba, Japan.
}

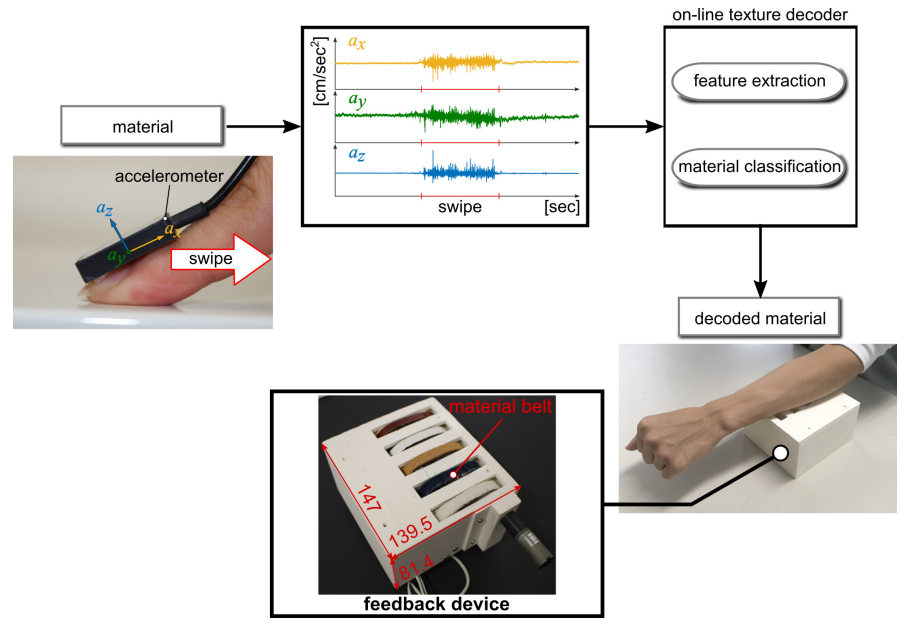

Fig. 1. Proposed tactile feedback system. The system consists of an accelerometer mounted on a fingertip. The features extracted from the accelerometer recordings were used to decode touched material using a linear sparse decoder. The decoded material was then presented to the user on the forearm of their left hand using the prototype direct-texture-feedback device.

texture information in scenarios of dynamic touch [11], [12]. Second, most studies on texture decoding have been performed in 'passive' experiments and dynamical texture explorative motions have hardly been studied in regard to texture feedback [13]. It is however known that passive external stimuli do not induce the natural tactile sensation [14]. Finally, almost all previous decoding studies have studied decoding independently from the actual feedback stimulation system it drives.

Vibratory stimulation has been the most popular substitutive feedback utilized for texture feedback due to the convenience of attaching a vibrator onto a subject's skin [15], [10]. However, adapting to a vibratory stimulation requires an user to learn new associations between vibratory parameters, such as frequency or intensity, and texture patterns.

In this study, we propose an on-line direct-texturefeedback system [16] that addresses the above issues. In line with previous studies that used vibration sensors for texture recognition [17], [18], [19], [20], [21]), our system uses a accelerometer based texture decoding system, but crucially we integrate it with a new 'direct-texture-feedback' device. The system enables on-line decoding of a texture touched by a user's finger or prosthetic limb, and provide feedback by choosing and presenting the actual material decoded, on another limb. The feedback device ensures material feedback at the speed at which a user swipes his finger, a feature that 
is known to improve texture perception [22].

In the following sections we describe our prototype system in detail. This is followed by the description of the two user studies to evaluate the performance of the decoding system as well as the feedback device. Finally, we discuss the results, and what we learned for the improvement of future devices. We also discuss the reason why we think directtexture decoding and feedback may be better than decoding and feedback of general texture characteristics like hardness or roughness.

\section{SYSTEM SETUP}

Our tactile feedback system consists of two parts, an accelerometer based decoding system, and a direct-texturefeedback system (Fig. 1). We describe each part in detail below.

\section{A. Decoding system}

The decoding system is an updated version of the method proposed in our previous study [23] using a finger or tool (simulating a prosthetic finger) mounted accelorometer and uses a sparse linear classifier [24] on the acceleromoter signals to decode/classify the the material the subject touches.

Followings are specific improvements incorporated in the current decoder, relative to the method from [23]:

a. Addition of a swiping motion detection for sampling only during explorative motions;

b. Addition of a swipe speed estimator utilizing the the accelerometer signals;

c. Utilization of new features in frequency domain, and estimated velocity and force of the active explorative motions, to improve decoding accuracy with fewer training samples, and over a larger range of swiping velocity.

1) Swiping motion detection: The data for decoding was extracted only for the period where we detected a 'finger swipe' (motion accompanied by touch). The swipe was detected using a threshold on the average acceleration change as:

$$
\sum_{i=1}^{m-1}\left|a_{n(i+1)}-a_{n(i)}\right| / m>T
$$

where $a_{n}=\sqrt{a_{x}^{2}+a_{y}^{2}+a_{z}^{2}}, m=200$ and threshold $T$ was equal to $10 \mathrm{~m} / \mathrm{sec}^{2} . a_{x}, a_{y}$, and $a_{z}$ are $\mathrm{x}, \mathrm{y}, \mathrm{z}$ component of the acceleration, respectively.

2) Swipe speed estimation: The swiping velocity $v$ at any time instant $t$ was estimated from the accelerometer as follows :

$$
v(t)=l \frac{\sum_{i=t-p}^{t-1} a_{x}(i)}{p}
$$

where $l$ is the frame length in msec, $p=200 / l$, and the $v$ is calculated with a length of $200 \mathrm{msec}$ at a window shift of $50 \mathrm{msec}$.
3) Decoding features: Our previous work utilized only frequency based features [23]. In this study we were able to improve decoder performance by including frequency and time domain features, as well as the information about the swipe velocity and swipe force. To calculate the frequency features, we considered both, the accelerations in the three axis as well as the three principle components of the accelerations in the three axis. From this we calculated $\left|\tilde{A}_{x}(f)\right|$, $\left|\tilde{A}_{y}(f)\right|$, and $\left|\tilde{A}_{z}(f)\right|$ are $\mathrm{x}, \mathrm{y}$, and $\mathrm{z}$ components of magnitude in frequency-domain after the Discrete Fourier Transform (DFT). This was used to calculate the 'vibration intensity' $V$ and the 'center frequency $f_{c}$ ' [17] :

$$
\begin{aligned}
V & =\sum_{f=100}^{1000} \log _{10}|\tilde{A}(f)|, \\
f_{c} & =\frac{\sum_{f=100}^{1000} f \cdot|\tilde{A}(f)|}{\sum_{f=100}^{1000}|\tilde{A}(f)|} .
\end{aligned}
$$

Each of these frequency-domain feature vectors was extracted from between a 100 to $1000 \mathrm{~Hz}$ frequency band, that is believed to include important signals for texture decoding. We calculated the vibration intensity and center frequency for the three accelerations as well as the three principle components.

We also calculated the equivalent DFT321 time signal [20] by performing the inverse Fourier transform of the frequency-domain magnitude of DFT321, where the DFT321 parameters $\left|\tilde{A}_{s}(f)\right|$ and phase $\theta(f)$ are defined as follows [20]:

$$
\begin{aligned}
\left|\tilde{A}_{s}(f)\right| & =\sqrt{\left|\tilde{A}_{x}(f)\right|^{2}+\left|\tilde{A}_{y}(f)\right|^{2}+\left|\tilde{A}_{z}(f)\right|^{2}} \\
\theta(f) & =\tan ^{-1}\left(\frac{\operatorname{Im}\left(\left|\tilde{A}_{x}(f)\right|+\left|\tilde{A}_{y}(f)\right|+\left|\tilde{A}_{z}(f)\right|\right)}{\operatorname{Re}\left(\left|\tilde{A}_{x}(f)\right|+\left|\tilde{A}_{y}(f)\right|+\left|\tilde{A}_{z}(f)\right|\right)}\right)
\end{aligned}
$$

As the time domain features, we used the root mean square and the average of the first order difference of the total acceleration $a_{n}$ and the equivalent DFT321 time signal.

To summarize, for the decoding we utilized 18 features-

- (vibration intensity $V$ and center frequency $f_{c}$ ) for the three components of acceleration and three principle components making 12 frequency features in total.

- The RMS (Root Mean Square) and average values of the total acceleration and DFT321 time signal, making four time features in total.

- the estimated swipe speed.

- the swipe force.

Each of these features were collected in 'samples' consisting of periods of $200 \mathrm{msec}$ collected over windows shifted by $50 \mathrm{msec}$. These features were ed through a sparse linear classifier to decode the swiped material [24]. 


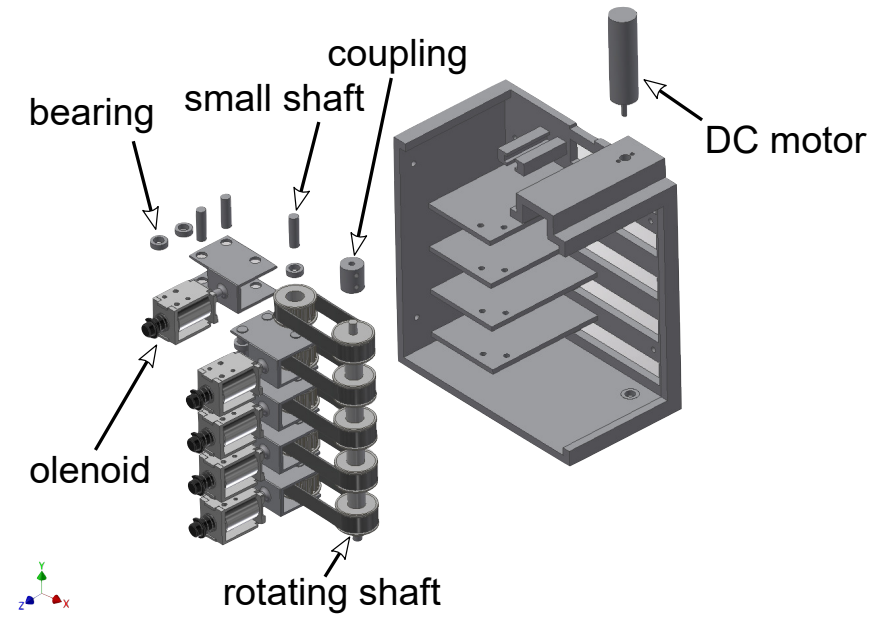

(a) disassembled

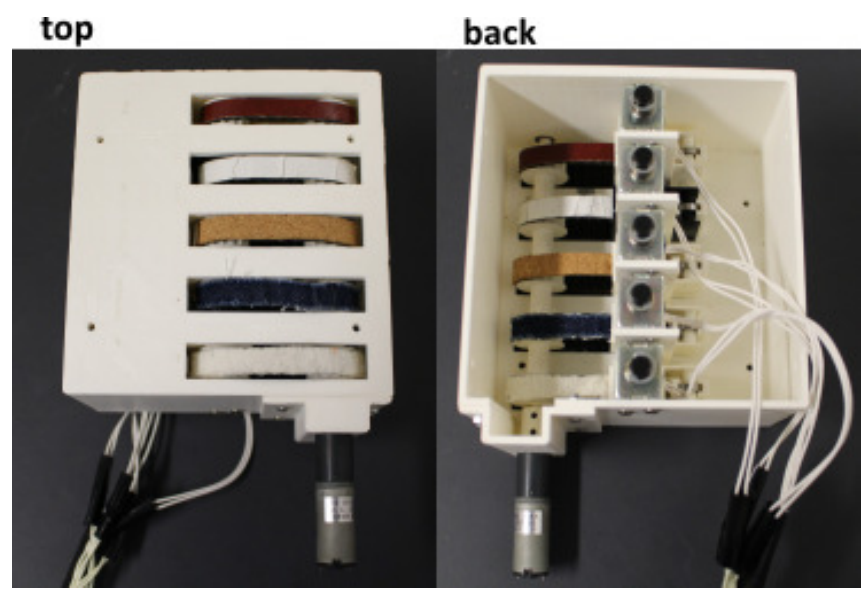

(b) assembled

Fig. 2. The direct-texture-feedback device. Our prototype device presents one of five material to the user. Each test material was fixed to the material belt with a tape. The material belts were attached to two pulleys and a DC motor was directly connected to the rotating shaft. A solenoid below each belt can be controlled to choose and lift one material to make contact with the user's skin. The speed and direction of the material contact was regulated by the DC motor to be equal to the swiping velocity of the user (calculated again from the accelerometer signals).

\section{B. Direct-texture-feedback device}

The second key contribution of this work is the development of a prototype low cost feedback device. This device was designed with the aim of presenting to the users, the actual materials that they touch with their pathological finger, or prosthetic finger (and which we recognize using the decoder). This direct-texture-feedback is presented on a different healthy part of the user's body.

In this first prototype, we developed a $3 \mathrm{~d}$ printed feedback device that is able to present one of five materials to the user by 'rubbing' them against the users skin on their forearm. The device uses a single motor and can adjust the speed of swipe on-line so as to match the velocity of their finger swiping the actual material (as recognized by our speed estimation), providing a sense of agency to the feedback.

The feedback device is shown in Fig. 2. The current prototype has medium dimensions (height $\times$ width $\times$ depth, $81.4 \times 139.5 \times 147.0 \mathrm{~mm})$ and is designed for feedback on the forearm of the user. The device consists of main rotating shaft with pulleys $(\phi 22 \mathrm{~mm})$. The main shaft is not actuated. A second rotating shaft is actuated by a DC motor (TG87A-GU-85.1-KA, TSUKASA ELECTRIC CO.,LTD.). Five belts made of different materials can be mounted side by side across the shafts, on the pulleys as shown in Fig. 2. Each belt measure $10 \mathrm{~mm}$ in width. Our device consists of five solenoid switches (CBS08300480, TAKAHA KIKO Co., Ltd.) placed between the shaft and the pulley, one each below a belt. The solenoids can be activated independently in order to push a belt up and rub against the skin of the subject. The solenoid is able to push the belt against the skin with a normal force of about $1.5 \mathrm{~N}$. The rated rotation velocity of the DC motor is $135.8 \mathrm{rpm}$, and is able to rotate the material belt at velocities between 1 to $10 \mathrm{~cm} / \mathrm{sec}$, velocities that have been shown to elicit comfortable tactile perception in a previous study [22].

\section{VERIFICATION EXPERIMENTS}

We conducted two experiments with participants to evaluate our device. The first experiment verified the scope of decoding performance of our updated decoder, to see what is the best performance one may expect from it. For this, we analyzed the decoder in a controlled training and testing scenario that enabled us to control, as well as measure, the speed and load of swipes. We tested the decoder for both the cases, when the swipe was made with the user's finger, and when the finger was artificial (and swiped by a user holding it).

Next, in the second experiment, we tested the tactile feedback system as a whole in the realistic user scenario in which a user swipes a material at his/her own comfortable speed, the material is decoded on-line during the touch, and the decoded results are fed back using our direct-texturefeedback device.

\section{A. Experiment 1: Verification of decoding performance}

a) Participants: Four healthy subjects (three males, one female, $22.8 \pm 1.0$ years old) participated in this experiment. The participants received explanation about all tasks before the experiments.

b) Experiment setup: The participants sat on a chair in front of a table. They were presented with one of five test materials (cork, denim, cotton, leather and paper) which they touched with their index finger (Fig. 3). A load sensor placed below the material allowed us to evaluate the force applied by the finger on the drum, that was read in through an Arduino microcomputer and then transmitting it to the PC through serial communication. A six-axis accelerometer (MPM6-06/2000C, Micro-Stone Corporation) was mounted on the finger tip of a participant. The accelerometer signals were read in using a multi-function DAQ device (USB6218, National Instrument). We also did sessions where the accelerometer was mounted on a $3 \mathrm{~d}$ - printed plastic finger, that a participant held in his hand and touched the test material- this simulated the prosthetic finger. 


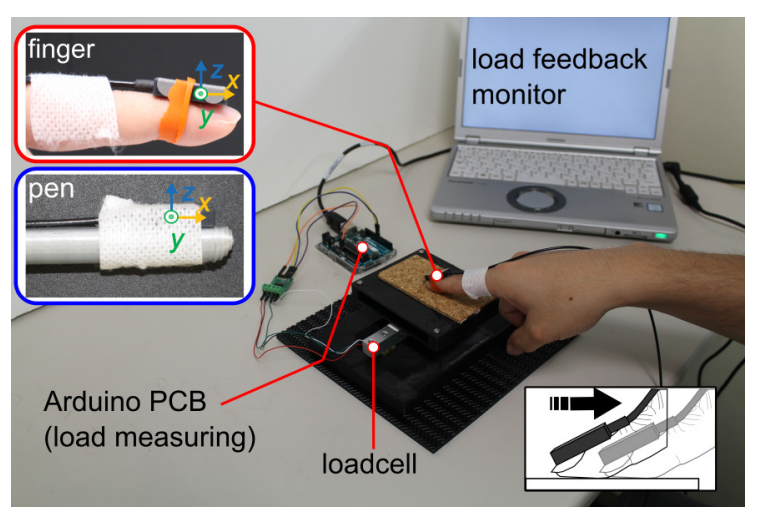

Fig. 3. Setup of Experiment 1. The photo shows the material plate (with a load cell below it) that the participants swiped either with their finger or while holding an artificial finger (see insets). They were presented with their swipe load feedback on a computer screen placed on the table in front of them.

c) Experiment procedure: The test materials were presented one at a time and the participant was asked to rhythmically swipe in a distal to proximal direction guided by rhythmic audio cues that were presented to them. The load applied to the test material was visually fed back to the participant on the monitor and they were asked to maintain the load near the target load displayed on the same monitor.

All measurements were done over a total of 30 conditions consisting of three target trial velocities $(10,20,30 \mathrm{~cm} / \mathrm{sec})$, two target loads $(0.49,0.98 \mathrm{~N})$, and five test materials. The accelerometer and load cell sampling rates were set at $4 \mathrm{kHz}$ and $20 \mathrm{~Hz}$, respectively.

The 30 conditions were repeated for the case when the same participant swiped the materials with the artificial finger.

d) Data pre-processing and decoder training: The accelerometer signal was Butterworth high pass filtered over $100 \mathrm{~Hz}$ before the features were evaluated. The swipe speed was Butterworth band passed between $0.125 \mathrm{~Hz}$ and $5 \mathrm{~Hz}$.

For each touch trial of a particular load and swipe speed, we collected 150 'training samples', that consisted of features (described in Sec.II-A.3) extracted from $200 \mathrm{msec}$ windows shifted by $50 \mathrm{msec}$. In total, in a total of 30 conditions (five materials $\mathrm{x}$ three swipe speeds $\mathrm{x}$ two swipe loads), we therefore recorded 4,500 samples. A decoder was constructed for each participant and trained with a total of 1500 samples, including 50 samples collected randomly from each swipe condition.

e) Decoder test results: The decoder for each participant was tested on 50 samples (that were independent of the training samples) collected randomly from all the conditions. We constructed five one-vs.-rest decoders (ex. cork-vs.-rest) from the data and the classification results of the decoder with the highest accuracy among the five provided us with the material decoding. This methodology was shown to be efficient in [23]. We performed this procedure 20 times to get the average performance for every participant. The across participant mean and standard deviation is shown as a confusion matrix in Fig.4a. This matrix shows what each

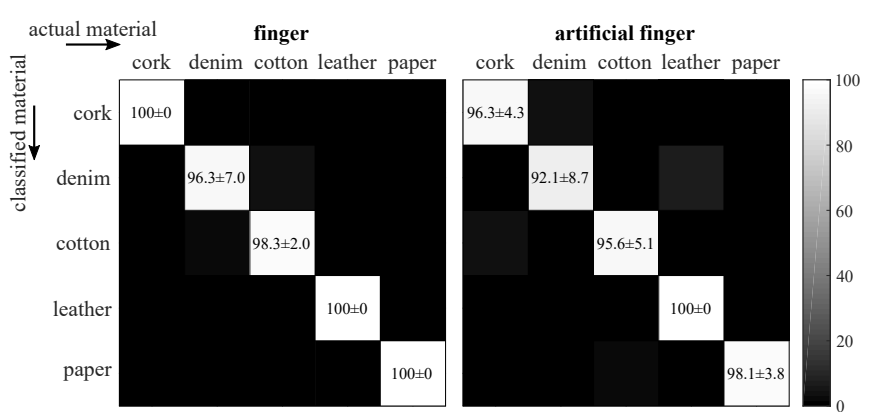

(a) with load feature

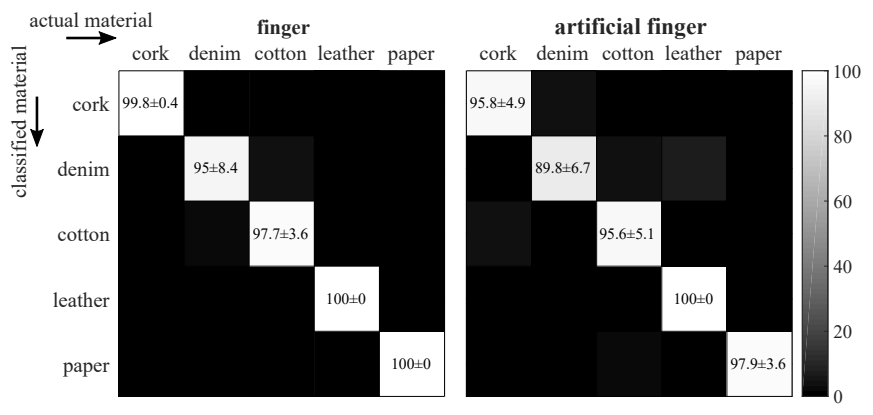

(b) without load feature

Fig. 4. Experiment 1, decoding accuracy. (a) The 'confusion matrix' presents the $\%$ mean and standard deviation decoding accuracy across participants- that is, what each presented test material (columns) was classified as (rows). The high diagonal values shows that the materials were decoded correctly for most participants. (b) The same results as a) when the swipe load features were omitted. We ound that the swipe load features do not influence the decoding accuracy significantly

test material (columns) was classified as (rows). Fig. 4a also shows the confusion matrix in the conditions when the swipe was done using the artificial finger. It was possible for the decoders to achieve decoding accuracies of more than $90 \%$ both, when the materials were touched with a participant's finger, and with an artificial finger.

Considering realistic scenarios (like the one we will test in Experiment 2), we also analyzed the decoding accuracy in the absence of a load sensor, hence omitting the swipe load as a feature (Fig.4b). We observed that even omitting the swipe load features, the decoder was able to achieve accuracies close to that with the load features. We therefore chose not use the load features in our next experiment.

\section{B. Experiment2: Verification of the decoder-direct-texture- Feedback system}

A texture identification experiment was conducted to show the efficacy of our direct-texture-feedback system. Specifically, considering a realistic application of the device for amputees, we tested, first, the effectiveness of the on-line implementation of our decoder, and second, the ability of users to identify the materials presented by our direct-texturefeedback device. In order for the participants to not identify the presented material directly from their swiping finger, we confined this experiment to swipes with the prosthetic finger (held by the participants in their hand). A foam padding on the prosthetic finger further ensured that the participants were 


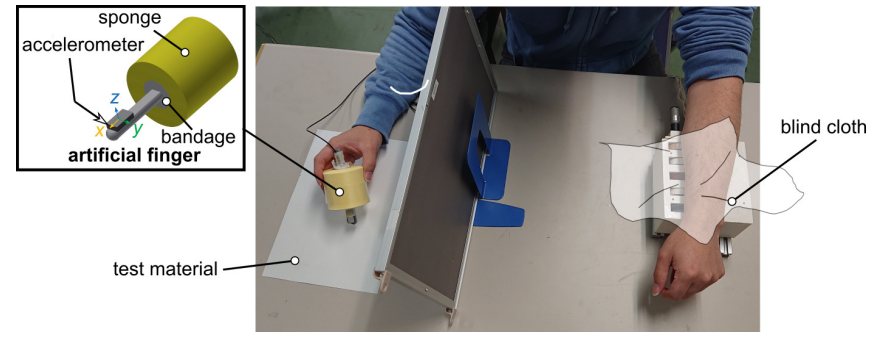

Fig. 5. Setup of Experiment 2. The test material was presented to the right hand of the participants while the feedback device was placed under the left forearm. The participants swiped the presented material with the artificial finger held in their right hand. A sponge surrounding the artificial finger prevented the participants from feeling the vibrations generated by the swipes. A board prevented the participants from seeing their right hand, while a piece of cloth covered the feedback device.

not able to feel (and hence distinguish) vibrations during the swipes.

a) Participants: Ten participants (eight males, two females, $26 \pm 0$ years old) participated in this experiment. All participants were healthy, without any known visual or tactile disabilities.

b) Experiment setup: During the experiment, participants held the prosthetic finger in their right hand, and sat in a chair in front of the monitor. They operated a 2-button button pad with their left hand. The subject was asked to swipe the tip of the artificial finger onto the test material surface $(200 \times 150 \mathrm{~mm})$ set in front of the right hand. The texture feedback device was placed below the left forearm of the participant (Fig. 5). The subject could adjust the position of their forearm during the experiment so as to optimize the perception of direct-texture-feedback.

Both the test material and the right hand were occluded from the participant's vision by a partition board. Furthermore, the subjects were blind folded with a piece of cloth. To avoid that the subjects identify the touched material from audio cues, they were asked to wear headphones that played white noise.

c) Experiment procedure \& On-line strategy: The subject was asked to swipe the test material rhythmically in a distal to proximal direction with the tip of the artificial finger at their 'preferred speed'. The feature vectors were extracted during the swipes in the same way as Experiment 1. The decoders were trained with 100 extracted samples.

The participants were presented with one randomly selected test material at a time. They swiped the material using the same sponge covered artificial finger. The online decoding was conducted by features extracted during the swipes, and the decoded material was presented to the participants using the direct-texture-feedback device. The participants were then presented with the name of materials one at a time on a computer screen in front to them. They used the button pad to answer if the presented material under their forearm and the screen were the same, or not. The materials were fed back by the direct-texture-feedback device in one of three speeds in random order- at the speed of the swipe ('normal'), at 67\% of the speed of their swipe ('slow')

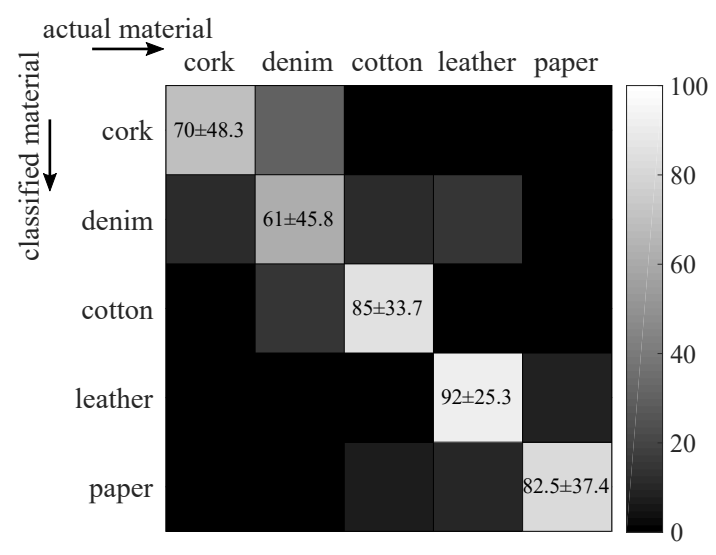

Fig. 6. Experiment 2, accuracy of the on-line decoder. The confusion matrix of the across participant $\%$ mean and standard deviation of the decoding accuracy for each presented material. The actual materials are shown along the columns, while the decoder classified material are shown along the rows.

and at $150 \%$ of the swipe speed ('fast').

d) Data analysis and results: The across participant means and standard deviations of the accuracy of the online decoder is shown in Fig. 6. Fig. 7 plots the material identification results at the different presentation speeds. Note that the identification results are calculated relative to the material presented to the participants, irrespective of the material actually touched by the participants.

We also analyzed the identification results in a two-way ANOVA, which evaluated two factors: the test materials (five materials, $d f=4$ ) and the speed of swipe (Slow vs. Normal vs. Fast, $d f=2$ ) and their interaction. We found that the materials did not affect the decoding accuracy $(\mathrm{F}(4,72)=0.55, p=0.69)$, but the accuracies changed depending on the speed of presentation $(\mathrm{F}(2,72)=4.72$, $p=0.02)$ and there was no interaction $(\mathrm{F}(8,72)=2.04, p$ $=0.06$ ). Across materials, post-hoc analysis showed that a significant difference was only observed between the fast and normal presentation $(\mathrm{T}(9)=2.32, p=0.045)$, but it did not survive multiple correction. These results suggest that a faster material presentation using the direct-texture-feedback device was better for identification, but further studies are required to verify this point.

\section{DISCUSSION}

\section{A. Summary}

In this study we developed a prototype texture feedback system including a on-line texture decoder and a directtexture-feedback device. The texture decoding in this system is achieved using a finger mounted accelerometer. We considered two cases- when the texture is swiped by a human finger (i.e when the device us used by a individual with sensory deficits), and when the texture is swiped by a artifical finger (i.e when the device is used by a amputee). A machine learning algorithm was trained to recognize the texture from the vibration signals recorded by the accelerometer when the fingers swiped a surface. 

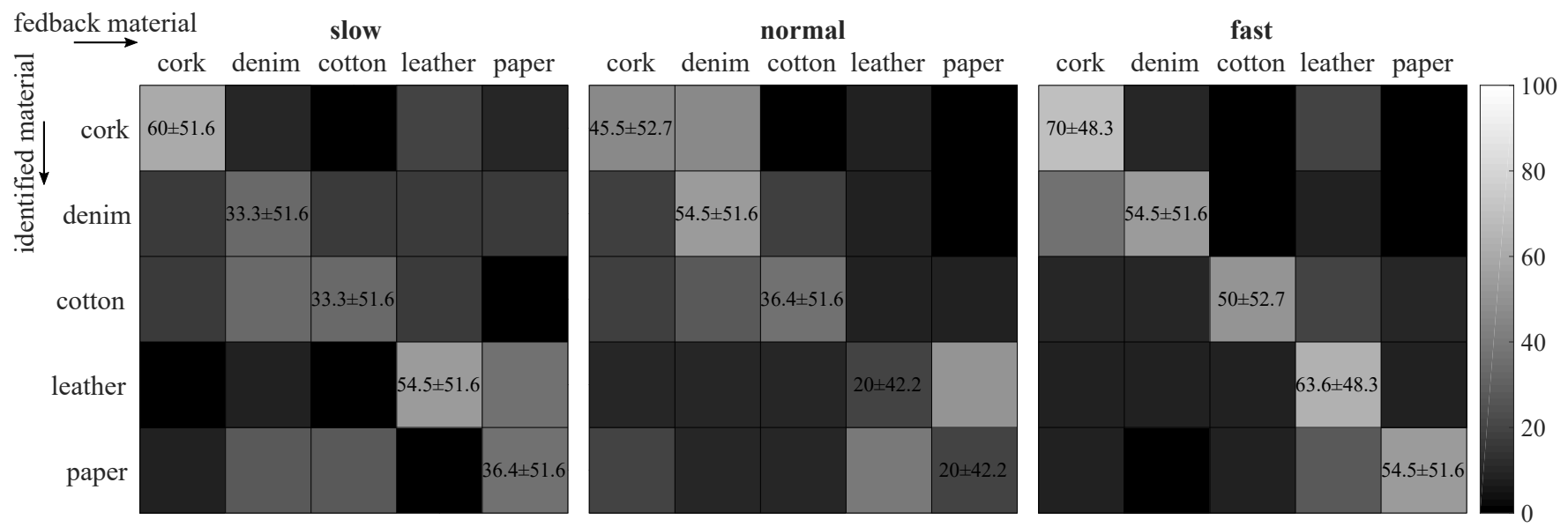

Fig. 7. Experiment 2, feedback perception by participants. The participants were presented with each test material in a random order which they swiped at their own preferred speed. The direct-texture-feedback device then presented the decoded material in one of three speeds- normal: at the speed of their swipe, slow: $67 \%$ of the swipe speed, and fast: $150 \%$ of the swipe speed. The confusion matrices show the (across participant) $\%$ mean and standard deviation of the materials identified by the participants (rows) corresponding to the materials presented to them (columns).

\section{B. Improving the on-line decoder}

We tested the decoder with five common materials and observed that the decoder could give excellent decoding accuracies of around 95\% correct recognition in an off-line decoding experiment (Experiment 1). In the on-line decoding (Experiment-2) however, this accuracy decreased to around $77 \%$ (Fig. 6). This is a significant decrease relative to the Experiment 1. We analyzed the reason for this and found that it was due to our flawed training methodology in Experiment 2. Unlike Experiment 1, in Experiment 2 we did not require the participants to swipe at different speeds during training, but let them swipe at their 'preferred speed'. We later found that, in the presence additional texture identification task, the variations in the participant speed was much higher, and they also consistently used higher swipe speeds during identification (Training: $4.39 \pm 3.09 \mathrm{~cm} / \mathrm{sec}$, Identification: $13.0 \pm 8.86$ $\mathrm{cm} / \mathrm{sec}$ ). In other words, their preferred speeds was very different in the decoder training and testing (identification task) phase, leading to attenuated decoder performance. We expect that training the decoder over a range of speeds (like in Experiment-1 should solve this problem and bring the recognition rates to the values achieved in Experiment 1.

\section{Lessons for direct-texture-feedback}

Once the texture being touched is decoded, the next step is to feedback the decoded texture to the user. Texture feedback is a non-trivial problem because the tactile perception of a texture is determined by not just the friction and the resulting vibrations induced by it, but also the modulated by the temperature of the material and the surface area of contact (for example a touched metallic surface makes contact only at the base of finger, while a fur coat will we make contact with not just the base but also the sides of a finger [19]). Hence many studies have opted to feedback just vibrations, assuming the user would learn the mapping between these and the material over time [15], [10]. Here we therefore opted for presenting directly the touched material (that we decode) as the feedback. In fact as the reader would have noticed, even our decoder is designed for a direct-texture-feedback device- we decode for specific material rather than some general property (like hardness, roughness, stickiness etc) of materials.

This option ofcourse ensures that the touch perception of the material (friction, temperature, contact area etc) are maintained during feedback, but on the other hand this means that we need to have all the material that a user may touch, 'in hand' and available for feedback. This seems like a impossible problem at first glance, but in fact the common material that we encounter in our daily life are not as many as one would imagine, especially if we group them into some general categories like metals, wood, cloth as so on.

In fact the relatively low identification rate in Experiment 2 (Fig. 7) showed that such catogorization may be in fact benefit texture feedback. We observed in Experiment 2 that users had difficulty distinguishing the materials presented to them, even when they knew that it was one of the five they had been shown before. This difficulty was not due to our choice of the feedback location. While in Experiment 2 we presented the feedback on their forearm, in preliminary studies, we found that users found identification difficult even when the feedback was provided near their shoulder, thigh or calf,or even the finger of their other hand (though performance in this case was improved a bit). This is maybe due to the fact that visual feedback is a crucial contributor for the identification of materials [25], [26]. However, in Fig. 7 , if we see the results of the Fast feedback (third panel) during which identification performance was best, we do see that users tend to mistake between certain material pairs more often. For example,denim is often mistaken as cork and vice-versa, and paper and leather are often mistaken as one another. The reason why these materials are perceived similar is unclear and needs further studies. However, these results suggest that feedback identification can be increased if the feedback is not of every material, but rather of material 
categories defined by the identification process. Such identification based categorization may also further help improve material decoder. We plan to test these issues in the next version of the device.

We conclude by noting that, in agreement with previous studies [22], we observed that the speed of feedback critically affected texture identification. First, the users reported that the correspondence between the material swipes and the feedback (enabled by our on-line velocity estimation) provided a feel of 'agency', making the feedback feel natural. Second, we found that the identification accuracy changed with the speed of feedback (Fig.7). Interestingly however, we found that the best accuracies were observed with a feedback that was 1.5 times faster than the user swipes. Further studies are required to understand the reason behind this and consequently, optimize the feedback speed.

\section{ACKNOWLEDGMENT}

KS and GG were partially supported by the JST ERATO Grant Number JPMJER1701, Japan.

\section{REFERENCES}

[1] L. M. Carey, T. A. Matyas, and L. E. Oke, "Sensory loss in stroke patients: Effective training of tactile and proprioceptive discrimination," Archives of Physical Medicine and Rehabilitation, vol. 74, no. 6, pp. 602-611, 1993.

[2] E. Ekstrand, J. Lexell, and C. Brogårdh, "Test-retest reliability of the Shape/Texture Identification testTM in people with chronic stroke," Clinical Rehabilitation, vol. 30, no. 11, pp. 1120-1127, 2016.

[3] A. Takagi, G. Ganesh, T. Yoshioka, M. Kawato, and E. Burdet, "Physically interacting individuals estimate the partner's goal to enhance their movements," Nature Human Behaviour, 2017.

[4] G. Ganesh, A. Takagi, R. Osu, T. Yoshioka, M. Kawato, and E. Burdet, "Two is better than one: Physical interactions improve motor performance in humans," Scientific Reports, 2014.

[5] L. Jiang, M. R. Cutkosky, J. Ruutiainen, and R. Raisamo, "Using haptic feedback to improve grasp force control in multiple sclerosis patients," IEEE Transactions on Robotics, vol. 25, no. 3, pp. 593-601, 2009.

[6] K. Kim and J. E. Colgate, "Haptic feedback enhances grip force control of sEMG-controlled prosthetic hands in targeted reinnervation amputees," IEEE Transactions on Neural Systems and Rehabilitation Engineering, vol. 20, no. 6, pp. 798-805, 2012.

[7] R. Davoodi and G. E. Loeb, "Development of a physics-based target shooting game to train amputee users of multijoint upper limb prostheses," Presence: Teleoperators and Virtual Environments, vol. 21, no. 1, pp. 85-95, 2012.

[8] K. Kita, Y. Otaka, K. Takeda, S. Sakata, J. Ushiba, K. Kondo, M. Liu, and R. Osu, "A pilot study of sensory feedback by transcutaneous electrical nerve stimulation to improve manipulation deficit caused by severe sensory loss after stroke," Journal of NeuroEngineering and Rehabilitation, 2013.

[9] E. K. Kim, K. B. Sugg, N. B. Langhals, S. M. Lightbody, M. E. Baltrusaitis, M. G. Urbanchek, P. S. Cederna, and G. J. Gerling, "An engineered tactile afferent modulation platform to elicit compound sensory nerve action potentials in response to force magnitude," 2013 World Haptics Conference, WHC 2013, vol. 22903, pp. 241-246, 2013.

[10] Z. H. Lin and S. Smith, "A natural user interface for realistic tactile perception of object surface texture," Proceedings - 9th International Conference on Intelligent Human-Machine Systems and Cybernetics, IHMSC 2017, vol. 2, pp. 370-373, 2017.

[11] A. Rana, J. P. Roberge, and V. Duchaine, "An Improved Soft Dielectric for a Highly Sensitive Capacitive Tactile Sensor," IEEE Sensors Journal, vol. 16, no. 22, pp. 7853-7863, 2016

[12] M. C. Jimenez and J. A. Fishel, "Evaluation of force, vibration and thermal tactile feedback in prosthetic limbs," in IEEE Haptics Symposium, HAPTICS, 2014.
[13] P. Galambos and P. Baranyi, "Vibrotactile force feedback for telemanipulation: Concept and applications," 2011 2nd International Conference on Cognitive Infocommunications, CogInfoCom 2011, 2011

[14] Y. Iwamura and M. Tanaka, "Postcentral neurons in hand region of area 2: their possible role in the form discrimination of tactile objects," Brain Research, 1978.

[15] M. R. Motamedi, J. P. Roberge, and V. Duchaine, "The Use of Vibrotactile Feedback to Restore Texture Recognition Capabilities, and the Effect of Subject Training," IEEE Transactions on Neural Systems and Rehabilitation Engineering, vol. 25, no. 8, pp. 1230-1239, 2017.

[16] E. Whitmire, H. Benko, C. Holz, E. Ofek, and M. Sinclair, "Haptic revolver: Touch, shear, texture, and shape rendering on a reconfgurable virtual reality controller," Conference on Human Factors in Computing Systems - Proceedings, vol. 2018-April, 2018.

[17] M. Natsume, Y. Tanaka, and A. Sano, "Skin-propagated vibration for roughness and textures," World Automation Congress Proceedings, vol. 2016-Octob, pp. 1-6, 2016.

[18] S. Ding, Y. Pan, M. Tong, and X. Zhao, "Tactile perception of roughness and hardness to discriminate materials by friction-induced vibration," Sensors (Switzerland), vol. 17, no. 12, 2017.

[19] R. Fagiani, F. Massi, E. Chatelet, Y. Berthier, and A. Akay, "Tactile perception by friction induced vibrations," Tribology International, vol. 44, no. 10, pp. 1100-1110, 2011.

[20] J. M. Romano and K. J. Kuchenbecker, "Creating realistic virtual textures from contact acceleration data," IEEE Transactions on Haptics, vol. 5, no. 2, pp. 109-119, 2012

[21] S. J. Bensmaia and M. Hollins, "The vibrations of texture," Somatosensory and Motor Research, 2003.

[22] L. S. Löken, J. Wessberg, I. Morrison, F. McGlone, and H. Olausson, "Coding of pleasant touch by unmyelinated afferents in humans," Nature Neuroscience, vol. 12, no. 5, pp. 547-548, 2009.

[23] K. Sakurada, G. Ganesh, W. Yu, and K. Kita, "Accurate Decoding of Material Textures Using a finger Mounted Accelerometer," in 2018 IEEE International Conference on Robotics and Biomimetics, ROBIO $2018,2018$.

[24] S. Yamamoto, S. Moizumi, and S. Kitazawa, "Referral of tactile sensation to the tips of L-shaped sticks," Journal of Neurophysiology, vol. 93 , no. 5, pp. 2856-2863, 2005

[25] S. J. Lederman and S. G. Abbott, "Texture perception: studies of intersensory organization using a discrepancy paradigm, and visual versus tactual psychophysics." Journal of Experimental Psychology: Human perception and performance, vol. 7, no. 4, p. 902, 1981.

[26] I. Kitahara, M. Nakahara, and Y. Ohta, "Sensory properties in fusion of visual/haptic stimuli using mixed reality," in Advances in Haptics. IntechOpen, 2010. 